\title{
Evaluating safety and compatibility of anti-tumor necrosis factor therapy in patients with connective tissue disorders
}

\author{
Jordan T. Said^, Scott A. Elman, Joseph F. Merola \\ Department of Dermatology, Brigham and Women's Hospital \& Harvard Medical School, Boston, MA, USA \\ Contributions: Conception and design: All authors; (II) Administrative support: JF Merola; (III) Provision of study materials or patients: None; (IV) \\ Collection and assembly of data: None; (V) Data analysis and interpretation: None; (VI) Manuscript writing: All authors; (VII) Final approval of \\ manuscript: All authors. \\ Correspondence to: Joseph F. Merola, MD, MMSc. Department of Dermatology, Brigham and Women's Hospital \& Harvard Medical School, 221 \\ Longwood Avenue, Boston, MA, USA. Email: jfmerola@bwh.harvard.edu.
}

\begin{abstract}
Inhibition of the proinflammatory cytokine tumor necrosis factor alpha (TNF $\alpha$ ) has been utilized as a treatment strategy for a variety of immune-mediated inflammatory disorders (IMID), including rheumatoid arthritis, Crohn's disease and psoriasis. A wide array of biologic therapies targeting the TNF $\alpha$ molecule, including etanercept, infliximab, certolizumab, golimumab and adalimumab, are routinely used in the care of patients with these conditions. In addition to their therapeutic potential, anti-TNF $\alpha$ agents commonly induce the formation of autoantibodies such as anti-nuclear antibodies and anti-double stranded DNA antibodies; however, the vast majority of these are of $\operatorname{IgM}$ isotype and of unclear clinical significance, uncommonly leading to drug-induced autoimmune disease. For these reasons, TNF $\alpha$ inhibition has been a controversial strategy in the treatment of primary connective tissue disorders (CTDs). However, as new therapeutics continue to be developed for the management of CTDs, the potential utility for anti$\mathrm{TNF} \alpha$ agents has become of great interest, demonstrated in several recent case series and small open-label trials. We review the safety and compatibility of anti-TNF $\alpha$ therapy in the management of systemic lupus erythematosus (SLE) and cutaneous lupus erythematosus (CLE), two well-studied example CTDs, as well as summarize the risks of autoantibody generation, infection, malignancy, and iatrogenic lupus flares as side effects of blocking TNF $\alpha$ in patients with these conditions.
\end{abstract}

Keywords: Tumor necrosis factor alpha (TNF $\alpha)$; systemic lupus erythematosus (SLE); cutaneous lupus erythematosus (CLE); connective tissue disease; autoantibodies

Submitted Jul 28, 2020. Accepted for publication Dec 31, 2020.

doi: $10.21037 /$ atm-20-5552

View this article at: http://dx.doi.org/10.21037/atm-20-5552

\section{Introduction}

Tumor necrosis factor alpha $(\mathrm{TNF} \alpha)$ is a proinflammatory cytokine produced by diverse immune cells: predominantly macrophages, but also lymphocytes, cutaneous mast cells, eosinophils, and natural killer cells (1-3). TNFa is responsible for the acute phase reaction during acute inflammation, inducing the release of C-reactive protein and other acute phase reactants. TNF $\alpha$ also works in parallel with interleukin-1 (IL-1) and interleukin-6 (IL-6) on the hypothalamus-pituitary axis to induce fever and as an immunomodulatory molecule to activate cells of the innate immune system (1).

The blockade of TNF $\alpha$ has emerged as an effective treatment strategy in the management of immune-mediated inflammatory disorders (IMID), best demonstrated in

$\wedge$ ORCID: 0000-0002-0357-6916. 
rheumatoid arthritis (RA) and Crohn's disease (4-7). Biologic therapies targeting the TNF $\alpha$ molecule, including etanercept, infliximab, certolizumab, golimumab and adalimumab, are routinely used in the care of patients with these conditions (8). However, TNF $\alpha$ blockade carries several risks that warrant consideration for patient safety. Anti-TNF $\alpha$ therapy often induces the formation of autoantibodies against nuclear antigens (ANAs) which are associated with a variety of primary autoimmune diseases and connective tissue disorders (CTDs); in addition, these agents carry a risk for drug-induced CTDs (9-12). As a result of these risks, the application of anti-TNF $\alpha$ therapy in the management of primary CTDs has been an active area of controversy.

Systemic lupus erythematosus (SLE) and cutaneous lupus erythematosus (CLE) represent classical autoimmune disorders in which to evaluate the safety and compatibility of anti-TNF $\alpha$ therapy in CTD. SLE is a heterogenous autoimmune disease with the potential for profound impact on quality of life and associated increased morbidity and mortality $(13,14)$. Presentations of SLE are heterogenous, but most commonly include mucocutaneous lesions, arthritis, renal inflammation, cytopenias, neuropsychiatric changes, and fever. Serologically, SLE is characterized by the presence of particular autoantibodies, some of which can be also be induced by anti-TNF $\alpha$ therapy. The antidsDNA and anti-Smith antibodies are the most specific for SLE, but other antinuclear antibodies may be present (15).

CLE is a diverse, heterogenous group of variants of lupus erythematosus that are limited to manifestations in the skin (16). Similarly to SLE, primary CLE has been associated with the presence of autoantibodies that can also be induced by anti-TNF $\alpha$ therapy, including ANA, antidsDNA, and anti-phospholipid (aPL) antibodies $(17,18)$.

\section{TNF $\alpha$ and anti-TNF $\alpha$ therapy in systemic, cutaneous, and drug-induced lupus}

In both mice and humans, TNF $\alpha$ has been demonstrated to be increased in the serum of subjects with SLE versus appropriate controls (2). Similarly, studies on human renal and cutaneous biopsies have shown TNFa levels and expression are elevated in SLE patients compared to control patients, and that the degree of elevation correlated with disease activity (19). This result suggests that TNF $\alpha$ expression in SLE may have a dose-like effect, with worsening disease associated with increased cytokine concentration. In addition, studies on refractory skin lesions biopsied from SCLE patients demonstrated a strongly positive epidermal distribution of $\mathrm{TNF} \alpha$ compared to control skin lesions, suggesting that $\mathrm{TNF} \alpha$ is implicated in the cutaneous pathogenesis of SCLE, as well (20).

More recent publications have used genetics methods to ascertain the definite link between TNF $\alpha$ and SLE. One experiment analyzed 204 Indian female SLE patients and 224 age- and sex-matched healthy controls to identify two specific single nucleotide polymorphisms (SNPs) in the promotor upstream of the TNFa gene on human chromosome 6 that are associated with an increased susceptibility to development of SLE (G-238A and G-308A) (21). Prior studies have demonstrated these two alleles to be associated with increased TNF $\alpha$ mRNA transcription. This study concluded that elevated plasma TNF $\alpha$ is observed in SLE patients and that these activating SNPs were more common in SLE patients, thus providing further evidence that TNF $\alpha$ is implicated in SLE pathogenesis.

As noted above, TNF $\alpha$ is implicated in the pathogenesis and symptomatology of SLE and CLE and would seem an obvious target in the treatment of these autoimmune conditions. However, despite increasing evidence that TNF $\alpha$ could be an efficacious drug target, the prospect of anti$\mathrm{TNF} \alpha$ therapy for lupus patients remains both interesting and highly controversial. This, in large part, is due to the concern for these medications' propensity to induce the disease states that they can theoretically treat, such as that described as the clinical entity anti-TNF $\alpha$-induced lupus erythematosus (ATIL) (9). This paradoxical behavior has been observed with anti-TNF $\alpha$ therapy and other diseases as well. For example, multiple studies have demonstrated the potential for anti-TNF $\alpha$ drugs to treat sarcoidosis, but also to induce drug-induced sarcoidosis $(22,23)$. Similarly, anti$\mathrm{TNF} \alpha$ agents have been shown to have utility in managing primary psoriasis, but reports of anti-TNF $\alpha$-induced psoriasis also exist (24-26). Finally, this phenomenon has been observed with dermatomyositis as well, with case reports and small case series demonstrating both treatment and induction with anti-TNF $\alpha$ agents $(27,28)$.

The diagnosis of ATIL is generally established when a clear temporal association exists between the onset of symptoms and the initiation or increased dosing of antiTNF $\alpha$ therapy. The symptoms must include at least one serologic (ANA or anti-dsDNA antibodies) and one nonserologic (various organ system manifestations) from the American College of Rheumatology criteria for primary SLE (12). Prior work has found that the anti-TNF $\alpha$ agents etanercept and infliximab appear to be slightly more 


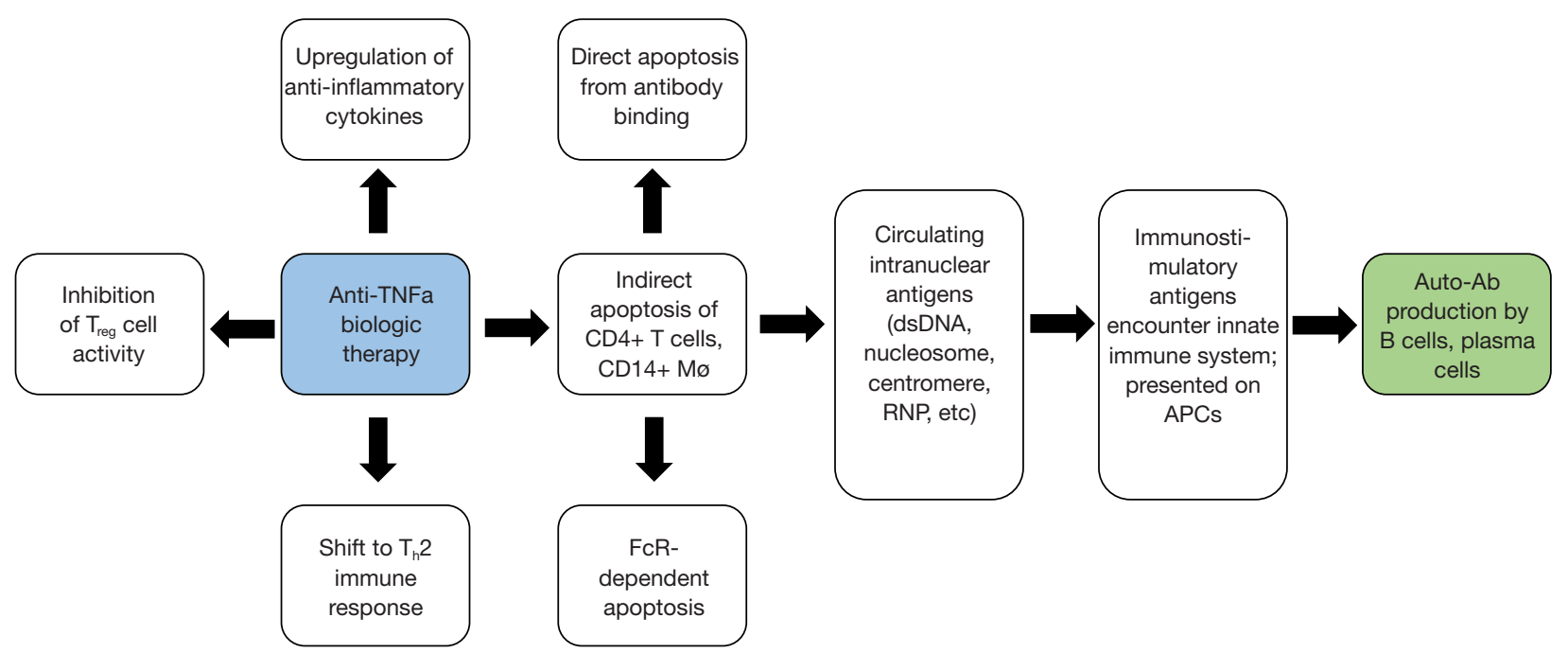

Figure 1 Pathogenesis of autoantibody induction by anti-TNF $\alpha$ biologic therapy. $\mathrm{T}_{\text {reg }}$, regulatory $\mathrm{T}$-cell; $\mathrm{TNF} \alpha$, tumor necrosis factor alpha; FcR, Fc receptor; dsDNA, double-stranded deoxyribonucleic acid; RNP, ribonucleoprotein; APC, antigen-presenting cells; Ab, antibody.

commonly associated with the induction of ATIL compared to adalimumab, although all three of these medications have been found to induce manifestations of SLE and likely represents a class-effect (9).

On the contrary, there is a growing body of literature demonstrating the efficacy and success of anti-TNF $\alpha$ therapy for CTDs, particularly SLE and CLE $(29,30)$. These works include individual case reports and case series, as well as several small completed and ongoing open-label trials from the past two decades. The analysis of several of these trials together supports the utility of anti-TNF $\alpha$ therapy for the management of complex CTDs, particularly in the resolution of lupus nephritis in SLE (discussed in greater detail in a later section) (31).

Herein, we review and discuss the evidence to date that supports the safety and compatibility of anti-TNF $\alpha$ therapies for patients with primary CTDs using SLE/ CLE as a model disease, with emphasis on autoantibody generation and organ damage secondary to drug-induced lupus. In each section, we review existing evidence for these risks that may generalizable from outside the SLE/CLE population, and then summarize existing literature on SLE and CLE patients managed with TNF $\alpha$ blockade.

\section{Autoantibody generation}

Much of the controversy surrounding the safety of antiTNF $\alpha$ therapies for SLE/CLE patients stems from the risk of inducing the formation of SLE/CLE-associated antibodies. Immunologically, these autoantibodies likely form as a result of anti-TNF $\alpha$-induced apoptosis and resulting circulation of immune-stimulatory nuclear antigens (Figure 1) (32-34). Furthermore, TNFa normally inhibits the production of type I interferon (IFN) from peripheral dendritic cells; anti-TNF $\alpha$ agents can thus shift the immune system toward type I interferon, which influences plasma cell differentiation and has been found to be upregulated in many IMIDs, including RA, SLE, and psoriasis (35). Encouragingly, prior data in RA patients showed that the induction of novel autoantibodies under $\mathrm{TNF} \alpha$ blockade was unrelated to type I IFN levels (36). However, this finding may not be consistent in patients with CTDs: among SLE patients for example, type I IFN serologies are known to be directly correlated with autoantibody induction (37). As a result, type I IFN upregulation secondary to anti-TNF $\alpha$ blockade may have more pathogenic consequence in SLE patients than in RA patients, owing to the encouragement of autoantibody generation.

Anti-TNF $\alpha$ therapies when used outside of the SLE/CLE population are well demonstrated to induce the formation of both ANAs and anti-dsDNA antibodies. With respect to ANAs, TNF $\alpha$ blockade can both elevate the titer of a patient with a baseline positive ANA serology and cause new-onset positive ANA in a previously negative ANA patient (38). In a meta-analysis of autoantibody generation amongst patients 
with RA or Crohn's disease treated with anti-TNF $\alpha$ therapy, infliximab induced ANAs in 29-76.7\% and anti-dsDNA antibodies in $10-29 \%$ of pooled patients; in comparison, etanercept for RA induced ANAs in $11-36 \%$ and antidsDNA in $5-15 \%$ of patients; adalimumab for RA notably only induced ANAs in $12.9 \%$ and anti-dsDNA in $5.3 \%$ of patients, based on a single study (4). This is consistent with other studies reporting that etanercept induces autoantibody formation somewhat less frequently than infliximab; adalimumab has a significantly reduced risk (11).

Key molecular and immunological differences exist between the anti-dsDNA autoantibodies generated in primary lupus erythematosus and the iatrogenic antidsDNA antibodies that occur secondary to anti-TNF $\alpha$ therapy. TNF $\alpha$ blockade-induced anti-dsDNA antibodies in RA patients have been shown to be of predominantly IgM class. This is opposed to primary SLE autoantibodies being predominantly of the IgG isotype. It is worth noting that anti-dsDNA isotype predominance is linked to clinical phenotype amongst SLE patients: one study demonstrated that higher levels of IgA anti-dsDNA $(\mathrm{P}=0.0002)$, antidsDNA $\operatorname{IgG} / \operatorname{IgM}(\mathrm{P}=0.001)$ and $\operatorname{IgA} / \operatorname{IgM}(\mathrm{P}<0.0001)$ ratios were associated with lupus nephritis, and that $\operatorname{IgG} / \mathrm{IgM}$ ratio $(\mathrm{P}=0.005)$ was significantly higher in patients with more active multisystem lupus disease (10). These findings collectively suggest that the presence of IgG anti-dsDNA antibodies carries a higher systemic pathogenicity compared to IgM anti-dsDNA, which has been proposed to in fact be nephroprotective (39). In addition, the more pathogenic anti-dsDNA IgG antibodies have been reported to develop in $\sim 0.5-1 \%$ of patients treated with anti-TNF $\alpha$ therapy; their presence is strongly associated with transient ATIL amongst patients with RA and Crohn's disease $(7,40)$.

It is paramount to note that much of the data on risks and adverse effects of anti-TNF $\alpha$ therapy was collected through studies on patients with RA, spondylarthritis, and Crohn's disease. There is a large absence in the literature and amongst clinical trials reporting safety information for these therapeutics amongst patients with CTDs. This is in part due to the rare reporting of CTD induction across anti-TNF $\alpha$ therapy clinical trials, likely related to its relative rarity compared to more commonly precipitated adverse events. More broadly, CTD patients are often excluded from clinical trials which could capture safety signals or efficacy data of TNF $\alpha$ blockade in these disease states. As a result, much of this discussion relies on small study populations, case series, and individual case reports.

An open-label safety study of seven patients with SLE who received four doses of $300 \mathrm{mg}$ infliximab over 10 weeks in addition to their baseline azathioprine or methotrexate led to an increase in the titer of anti-dsDNA IgG in five of the six patients with preformed antibodies; an increase in serologic titer was first noted to be significant at week 10 at the time of final infusion, and peaked four weeks later at over twice the patients' baseline values, at which time infliximab should not have totally cleared from the patients' serum (41). Interestingly, amongst SLE patients, the titer or mere presence of pre-formed anti-dsDNA autoantibodies may impact the risk of autoantibody generation on antiTNF $\alpha$ therapy: in this study, the single patient who did not have pre-formed anti-dsDNA antibodies did not demonstrate seroconversion to positive titer for antidsDNA antibodies over the duration of the study (41).

This elevation of anti-dsDNA antibodies in SLE patients by anti-TNF $\alpha$ therapy raises safety concerns for downstream clinical effects of this particular SLE-associated antibody. In particular, multiple studies have directly identified anti-dsDNA as pathogenic in proliferative lupus nephritis, with evidence that kidney biopsy elution from SLE nephritis patients show elevated anti-dsDNA and that injection of anti-dsDNA into murine models causes lupus nephritis-like histopathologic changes $(42,43)$. Elevated serology of anti-dsDNA antibodies has been associated with a clinical risk for lupus nephritis (44). However, in this study of six SLE patients who received infliximab, none of the patients demonstrated disease flares while receiving $\mathrm{TNF} \alpha$ blockade, regardless of baseline anti-dsDNA serology or increase in titer during the study (41). In fact, a second open-label safety study of infliximab for SLE demonstrated significant efficacy for lupus nephritis, with proteinuria decreasing significantly after one week and remaining at low levels for multiple months after cessation of infliximab (45). These studies together consistently and convincingly suggest that while anti-dsDNA IgG antibodies titers can increase in SLE patients on TNFa blockade-possibly more likely in those with preformed antibodies-patients typically demonstrate a stable improvement in proteinuria, rather than experience an ATIL-like disease flare with a glomerulonephritis component.

This finding suggesting the benevolence of the increase in anti-dsDNA antibody titer in SLE patients under TNF $\alpha$ blockade is supported by further recent studies. A multi-center study of patients with coexistent psoriasis/ psoriatic arthritis and SLE/CLE found that of 20 patients that received etanercept or infliximab, six of these patients seroconverted to positive ANA or anti-dsDNA serologies; 
however, these seroconversions proved to be subclinical, as no lupus flares occurred in these patients (26). Interestingly, a possible flare of primary lupus was observed in one of these 20 patients, who did not experience seroconversion. This finding suggests that the induction of ANA or anti-dsDNA autoantibodies by anti-TNF $\alpha$ agents is not pathogenically linked to lupus flares in SLE/CLE patients (26).

Other autoantibodies are worth consideration with $\mathrm{TNF} \alpha$ blockade, though with less demonstrated evidence of associated clinical manifestations. The presence of antiphospholipid antibodies (aPLs) - including the lupus anticoagulant and anti-cardiolipin (aCL) autoantibodiesis associated with the clinical features of antiphospholipid syndrome (APS). Antiphospholipid antibodies generated secondary to anti-TNF $\alpha$ therapy have been demonstrated in non-SLE and SLE patients. Antiphospholipid antibodies have been detected in RA patients receiving $\mathrm{TNF} \alpha$ blockade, but have not been well linked to clinically significant manifestations across studies (6). A possible link may exist between bacterial infection while under TNF $\alpha$ blockade and aCL autoantibody induction: Ferraccioli et al. studied eight RA patients receiving etanercept and found aCL IgG induction in five patients, temporally associated with either $S$. aureus nasobronchial infection or E. coli urinary tract infection (UTIs); of note, appropriately targeted antibiotic therapy resulted in a rapid decline of aCL IgG to normal in these patients (46).

Antiphospholipid antibodies commonly develop in SLE patients on TNF $\alpha$ blockade, with few clinical manifestations. In the aforementioned 2004 study by Aringer et al., 4/6 moderately active SLE patients on infliximab and either azathioprine or methotrexate showed increased aCL titers; however, this was not associated with a decrease in serum complement, or an increase in vascular events or lupus flares (45). A second study in 2007 showed that 4/7 of SLE patients treated with infliximab demonstrated aCL IgM titers temporally associated with TNF $\alpha$ blockade; none of the patients experienced thrombotic or thromboembolic events, and aCL titers decreased after a peak no later than 10 weeks after therapy was stopped (41). These studies, while small, suggest that aCL antibodies induced or increased in SLE patients on TNF $\alpha$ blockade do not appreciably increase the risk for thrombotic events or other clinical manifestations.

Despite these reports, two cases suggest that vascular events secondary to aPL in SLE patients are possible. In a long-term follow-up study of the two aforementioned cohorts of six and seven SLE patients treated with infliximab, one patient who had a transient increase in aCL IgG while receiving infliximab went on to develop a deep vein thrombosis (DVT) 14 weeks after receiving their fourth and final infliximab infusion, shortly after the aCL titer returned to baseline (31). Anticoagulation successfully managed this patient, who was well without complications 30 months later. Furthermore, in a study of nine patients with refractory lupus nephritis treated with three doses of infliximab, one patient suffered a lethal brainstem infarction three months after the final infliximab infusion (47). Of note, this patient had no detectable aPL or lupus anticoagulant titer at time of death, but a prior review article has noted that the vascular event may still theoretically have been related to aPL antibodies (8).

In summary, the induction of aPLs amongst SLE patients receiving anti-TNF $\alpha$ therapy is only rarely associated with adverse events. However, while DVT and stroke were uncommon events, the seriousness of these conditions warrants concern with the induction of aPL antibodies. Baseline risks for venous thrombosis and arterial vascular events should be considered in patients who develop these autoantibodies under TNF $\alpha$ blockade.

Finally, a prior analysis of a subset of patients' autoantibody profiles showed that all SLE patients treated with infliximab demonstrated an increase in autoantibodies against nuclear antigens, particularly specific anti-histone and anti-chromatin antibodies, without clinically correlated effects that returned to baseline follow treatment cessation (41).

\section{Lupus disease flares}

It is well known that anti-TNF $\alpha$ therapy can induce the aforementioned ATIL (9). The concern for anti-TNF $\alpha$ biologics to worsen or induce SLE or CLE-associated symptoms has evoked major concern for this therapeutic approach in SLE/CLE patients, as well as in CTD patients at large. Three clinical syndromes have been proposed as theoretical risks of TNF $\alpha$ blockade in SLE/CLE patients: drug-induced lupus erythematosus (DILE) caused by nonTNF $\alpha$-blocking drugs, the more specific syndrome ATIL, and flares of primary SLE/CLE disease $(8,9,48)$.

Prior reviews have made immunological and symptomatic distinctions between DILE caused by non-TNF $\alpha$-blocking drugs and the more specific ATIL. As discussed in the previous section, anti-TNF $\alpha$ therapy has been demonstrated to induce and elevate a wide array of lupus-associated antibodies. The presence or increase in titer of anti-histone antibodies is not pathognomonic for DILE, but anti-histone 
antibodies are present in $75-95 \%$ of DILE cases, varying across study populations $(49,50)$; other studies report their presence in only $17-57 \%$ of ATIL $(12,51)$. Early papers hypothesized that low-affinity anti-dsDNA IgM and IgA antibodies were implicated in the pathogenesis of DILE (6). In contrast, anti-dsDNA IgG antibodies may be pathogenic in ATIL: as mentioned, $0.5-1 \%$ of RA and Crohn's disease patients have been show to develop new anti-dsDNA IgG autoantibodies under TNF $\alpha$ blockade, strongly associated with the development of ATIL in these patients $(7,40)$. Renal disease is rare in DILE while common in ATIL, and antidsDNA IgG have been associated with lupus nephritis (51). Finally, flares of ATIL have been shown to have a higher prevalence of positive anti-dsDNA antibody titer, cutaneous eruptions, and hypocomplementemia (51). Both DILE and ATIL would be expected to remit in the weeks after therapy was ceased, although complete resolution may take up to several months in rare cases (52). Autoantibody titers fall more slowly than symptoms vanish following cessation of the inciting drug, and can remain elevated for several months to years (52).

Significantly, Aringer et al.'s first open-label study found that anti-dsDNA IgG titers increased in 5/6 SLE patients treated with infliximab (45); the accompanying long-term follow-up study demonstrated that these titer elevations were quite common after infusions and that no flares of primary disease occurred across 13 patients (31). Thus, while supposed pathogenic autoantibody induction occurred, there was a total absence of corresponding clinical symptoms, with efficacy data showing a robust, stable decrease in proteinuria with $\mathrm{TNF} \alpha$ blockade (31).

In a study of seven SLE patients treated with infliximab, the amount of anti-histone antibodies measured by enzymelinked immunosorbent assay and immunoblotting was increased in 4/7 patients (41). There were no clinical manifestations consistent across these four patients. None of the seven patients demonstrated symptomatic lupus flares. Across both of these short-term studies, no patients were observed to exhibit flares of lupus-associated symptoms. In addition, Aringer et al.'s long-term follow-up of 13 SLE patients treated with infliximab found no episodes of primary disease flares, including novel organ manifestations or hypocomplementemia (31). These data suggest that TNF $\alpha$ blockade may 'unlink' autoantibody induction from flares of iatrogenic disease, as the elevation of anti-histone and anti-dsDNA antibody titers were not associated with flares of DILE or ATIL, respectively.

These studies are consistent with Varada et al.'s findings in coexistent psoriasis/psoriatic arthritis and SLE/CLE (26). Among 20 patients with these concomitant conditions treated with anti-TNF $\alpha$ biologics, just one patient was found to have what could only be qualified as an unclear, possible lupus flare. This patient had a 14-year history of SLE with flares predating biologic therapy, and developed class IV glomerulonephritis 60 days after treatment with infliximab. The flare did resolve with discontinuation of infliximab, which could be consistent with ATIL or primary lupus flare; however, the patient experienced a second flare 15 months after discontinuation, which may instead suggest insufficient management by infliximab (26). In summary, we thus note that there was no strong evidence across these studies suggestive of flares of primary SLE disease while on TNF $\alpha$ blockade $(26,31,41,45)$.

Of note, while these studies suggest the minimal-to-no risk of lupus flares in SLE patients, early reports suggest that anti-TNF $\alpha$ therapy may have a greater propensity to worsen existing disease in the CLE population. We discuss these findings later.

\section{Infectious risk}

Biologics targeting TNF $\alpha$ has been associated with an increased risk for opportunistic infections amongst patients without CTDs (53). TNF $\alpha$ plays an important role in macrophage activation and granuloma formation, and thus $\mathrm{TNF} \alpha$ blockade increases the risk for tuberculosis (TB) and other granulomatous infections (54). Specifically, the relative risk for TB increases by 1.6-25 times under TNF $\alpha$ blockade, depending on the specific disease, biologic agent, and patient country of origin (55). TNF $\alpha$ blockade also impacts phagosome formation and the antiviral immune response, which in non-SLE/CLE patients increases the risk for infection by intracellular bacteria and fungi as well as dormant viruses (i.e., HBV, VZV) (54).

It is paramount to note that SLE patients at baseline are more susceptible to non-severe and severe infections and have an increased mortality risk from acquired infections (56). Prior studies in RA patients have suggested that the maximum risk for serious infection is within the first 90 180 days of TNF $\alpha$ blockade $(57,58)$, which may be similarly relevant to CTD patients.

Both non-severe and severe infections have been reported in SLE patients managed with anti-TNF $\alpha$ therapy. Bacterial infections-particularly pneumonia and UTIs-have been most observed (8). Amongst three reported cases of bacterial pneumonia, one progressed to a fatal Legionella pneumonia 
$(31,59,60)$. Uncomplicated UTIs were particularly common across multiple small study populations of SLE patients treated with varying courses of infliximab, occurring as frequently as $3 / 6$ patients in one pilot study $(45,47,59)$. All three patients reported a past history of similar infections; none of these cases were associated with leukopenia or hypocomplementemia (suggesting primary lupus flare or ATIL), or required treatment cessation $(31,45)$. Interestingly, prior authors have hypothesized that the notable tendency for SLE patients under TNF $\alpha$ blockade to suffer UTI may be due to concentration of anti-TNF $\alpha$ biologics in the urinary tract owing to baseline proteinuria (8).

Finally, two non-lethal cases of Salmonella enteritis and molluscum contagiosum abscess have been reported (31). Both infections occurred within six months after receiving the final dose of infliximab. In summary, most infections reported thus-far were reassuringly non-severe, but at least one lethal infection has occurred.

\section{Malignancy risk}

The change in risk of solid or hematologic malignancyparticularly lymphoma-associated with anti-TNF $\alpha$ therapy is an active area of controversy, amongst all patients and patients with CTDs. One recent pooled study used data from all available placebo-controlled studies of TNF $\alpha$ targeted biologics in inflammatory bowel disease (IBD) and found that anti-TNF $\alpha$ therapy was not associated with an increased risk of malignancy in these patients within one year of treatment (61). More recently, a systematic review of previously published randomized controlled trials, observational studies, case series, reviews, and meta-analyses from 2000 to 2015 found no increased risks of breast cancer, lymphoma, or non-melanoma skin cancer were found in RA patients, and no increased risk of overall cancer was found in IBD patients (3).

Patients with CTDs-including SLE—have an increased baseline risk of lymphoma $(31,62,63)$. As stated previously, studies of patients with CTDs managed with TNF $\alpha$ blockade have been few and small in size, which reduces the power of an analysis to assess for increased malignancy risk in CTD patients under TNF $\alpha$ blockade, compared to baseline CTD patients or patients receiving TNFa blockade for other disease states.

Nonetheless, two reports of malignancy in SLE patients potentially associated with anti-TNF $\alpha$ therapy exist in the literature, including one case of central nervous system B-cell lymphoma which was diagnosed after 16 infliximab infusions, and one case of renal cell carcinoma 4.5 years after receiving infliximab $(31,62,63)$. Neither of these patients' malignancies could be causally related to TNF $\alpha$ blockade with certainty. At this time, given limited numbers of studies in SLE/CLE populations, we are unable to ascertain an altered risk of malignancy in these patients on anti-TNF $\alpha$ therapy.

\section{On the horizon: anti-TNF $\alpha$ therapy in CLE}

TNF $\alpha$-targeting biologics have also been demonstrated to cause forms of CLE as adverse events. Specifically, druginduced SCLE and, less commonly, discoid lupus, has been reported. This risk of TNF $\alpha$ blockade has made the use of these agents to manage primary CLE disease a controversial topic, given this paradoxical CLE risk.

Of note, anti-TNF $\alpha$ therapy can induce other cutaneous connective tissue disorders and autoimmune diseases, which may have parallels to CLE. Recent literature has reported on anti-TNF $\alpha$-induced dermatomyositis (DM), an autoimmune disease targeting the skin and muscles (27). Just as in CLE, evidence exists to suggest that the use of anti$\mathrm{TNF} \alpha$ agents in DM can exacerbate underlying disease (28). In addition, anti-TNF $\alpha$-induced $\mathrm{DM}$ demonstrates the importance of considering anti-TNF $\alpha$-induced CTDs versus generalized drug-induced CTDs. A case series of four patients with anti-TNF $\alpha$-induced DM remarked that these patients required corticosteroids and immunosuppression for optimal management, whereas drug-induced DM is generally improved by simply discontinuing the inciting agent (27).

Outside of the CTD population, syndromes resembling both SCLE and DLE have been reported in case reports and series to have been induced by etanercept $(5,64)$, infliximab $(65,66)$, and adalimumab $(67,68)$. Unlike in SLE, a single case report demonstrates a concern for SCLE flare after TNFa blockade. Wilkerson et al. described a 66-year-old woman with a past history of drug-induced SCLE originally caused by hydrochlorothiazide and furosemide. The patient was treated with subcutaneous injection of golimumab for RA and suffered a cutaneous photo-distributed eruption of scaly, annular, erythematous plaques on the arms, legs, and upper trunk 2-3 weeks later. Serology showed a positive ANA and elevated anti-La/SSA and anti-Ro/SSB antibody titers; punch biopsy was consistent with SCLE. This report demonstrates that a TNF $\alpha$-targeting biology has the potential to exacerbate underlying SCLE disease, and may have cross-reactivity with other drugs known to commonly 
induce forms of drug-induced CLE (69).

Despite the possible propensity for TNF $\alpha$ blockade to cause forms of CLE, three examples exist that paradoxically suggest early utility and mixed safety of using anti-TNF $\alpha$ biologics to treat forms of CLE. First, amongst Varada et al.'s study of psoriasis/psoriatic arthritis and lupus erythematosus included two patients with DLE (26). Both patients at the time of study were managed with ongoing etanercept, for 12 and 132 months, for management of psoriatic disease. Neither patient experienced seroconversion or adverse events requiring discontinuation of anti-TNF $\alpha$ therapy, including flares of DLE.

Second, a single case report in a case series from 2006 highlighted a 42-year-old female patient with both polymyositis and poorly controlled SCLE with pruritus who had not achieved clearance of lesions on methotrexate, hydroxychloroquine, and prednisone (70). The patient was treated with biweekly subcutaneous injections of 25 mg etanercept, which after three doses caused a fever and flare of SCLE, with biopsy consistent with SCLE and no evidence of drug eruption. The patient was rechallenged with etanercept and the SCLE flare did not occur with following administrations. The patient achieved clearance after three months; prednisone was tapered and hydroxychloroquine was discontinued. This patient notably had a history of recent SCLE flares predating TNF $\alpha$ blockade, and given the lack of subsequent flares after rechallenge, it is possible that her flare after three doses of etanercept was unrelated to TNF $\alpha$ blockade. No other adverse events were reported, demonstrating efficacious and otherwise safe use of etanercept for SCLE management.

Lastly, an ongoing clinical trial for the injection of etanercept into DLE lesions has shown promising early results. In a phase IIb trial, authors aimed to circumvent the risks of systemic TNF $\alpha$ blockade by injecting weekly low-dose etanercept into symptomatic DLE lesions in 25 patients to induce remission (71). Fifty-two percent of patients $(13 / 25)$ met the primary endpoint of at least $20 \%$ reduction of the injected lesion after 12 weeks of therapy. Although the authors noted detectable etanercept in serum in $25 \%$ of patients, the therapy was tolerable and no significant safety signals were observed. Given that TNF $\alpha$ is known to be enriched in the epidermis of symptomatic CLE lesions (20), this phase IIb trial may provide a novel solution by locally blocking TNF $\alpha$ while avoiding systemic risks of infused anti-TNF $\alpha$ therapy. It also demonstrates partial treatment of a primary CTD with TNF $\alpha$ blockade.

Compared to the small but successful trials demonstrating the efficacy of anti-TNF $\alpha$ agents for SLE patients, there is a significant lack of case series or small studies trialing the use of TNF $\alpha$ blockade in CLE. We await the results of ongoing and novel studies in this area to clarify the risks and safety of anti-TNF $\alpha$ biologics in CLE patients, but remark that an early trial suggests that TNF $\alpha$ inhibition can achieve at least partial treatment of primary CTD.

\section{Conclusions}

Due to both the continued uncertainty of the precise role of $\mathrm{TNF} \alpha$ in the pathogenesis of lupus erythematosus and longstanding association of TNF $\alpha$-targeting biologics with a risk for inducing CTD-like syndromes, physicians have hesitated to explore the utility of TNF $\alpha$ blockade for the management of CTDs, including SLE/CLE. Though existing studies are small in quantity and size, we have reviewed the known literature to date to summarize the overall safety and compatibility of anti-TNF $\alpha$ therapy for treating relevant indications (e.g., psoriasis, IBD), among patients with concurrent SLE/CLE (Tables 1,2). We note that autoantibodies (such as anti-dsDNA) are commonly elevated by TNF $\alpha$ blockade in SLE patients, but are most typically non-pathogenic IgM. In fact, potential benefit among SLE patients with nephritis has been broadly demonstrated as a stable decrease in proteinuria rather than a flare of primary disease or glomerulonephritis. In contrast, aPL autoantibodies were commonly elevated and rarely associated with severe vascular events (DVT, infarction). Evidence does not support the exacerbation of primary SLE by anti-TNF $\alpha$ therapy. Finally, we note that early data suggests the possible utility of anti-TNF $\alpha$-therapy for partial treatment of CLE, a model for CTD. We await further studies investigating the utility and safety of these agents. 
Table 1 Summary of risks of anti-TNF $\alpha$ therapy in SLE/CLE patients

\begin{tabular}{ll}
\hline Risk of anti-TNF $\alpha$ therapy & Appraisal of existing evidence \\
\hline Autoantibody formation & $\begin{array}{l}\text { Anti-dsDNA IgG antibodies titers can increase in SLE patients on TNF } \alpha \text { blockade, but patients typically } \\
\text { demonstrate a stable improvement in proteinuria, rather than experience a flare of primary disease or } \\
\text { glomerulonephritis. Patients with SLE receiving anti-TNF } \alpha \text { therapy rarely suffer aPL-associated vascular adverse } \\
\text { events (DVT, infarction), thus warranting concern if the induction of aPL antibodies occurs }\end{array}$ \\
Primary disease flares & $\begin{array}{l}\text { No evidence in reviewed studies of SLE patients is suggestive of flares of primary SLE disease while on TNF } \alpha \\
\text { blockade }\end{array}$ \\
Infection & $\begin{array}{l}\text { SLE patients, who at baseline have a higher risk of infection, treated with TNF } \alpha \text { blockade may suffer from minor } \\
\text { infections, such as UTI. The risk of severe infection appears to be low, but lethal bacterial pneumonias have } \\
\text { been reported } \\
\text { Given limited numbers of studies in SLE/CLE populations, we are unable to ascertain an altered risk of } \\
\text { malignancy, including lymphoma, in these patients on anti-TNF } \alpha \text { therapy }\end{array}$
\end{tabular}

dsDNA, double-stranded deoxyribonucleic acid; TNF $\alpha$, tumor necrosis factor alpha; SLE, systemic lupus erythematosus; aPL, antiphospholipid; CLE, cutaneous lupus erythematosus; TB, tuberculosis; DVT, deep vein thrombosis.

Table 2 Pros and cons of anti-TNF $\alpha$ therapy in SLE/CLE patients

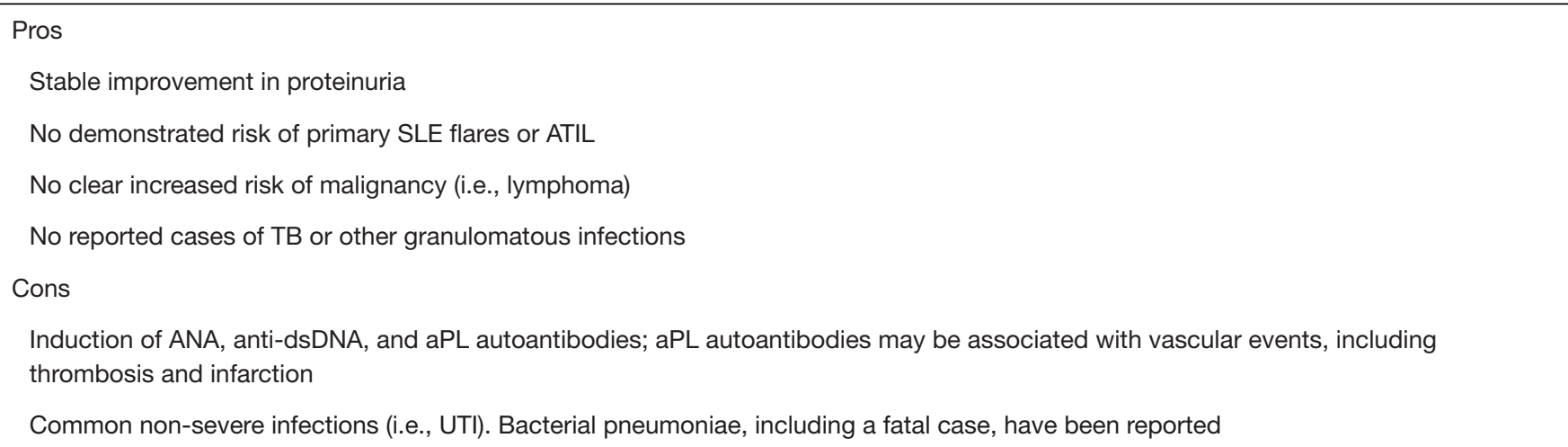

TNF $\alpha$, tumor necrosis factor alpha; SLE, systemic lupus erythematosus; dsDNA, double-stranded deoxyribonucleic acid; aPL, antiphospholipid; ATIL, anti-TNF $\alpha$-alpha-induced lupus erythematosus; UTI, urinary tract infection; TB, tuberculosis.

\section{Acknowledgments}

Funding: None.

\section{Footnote}

Provenance and Peer Review: This article was commissioned by the Guest Editors (Drs. Richard D. Sontheimer, M. Kari Connolly, David F. Fiorentino, and Victoria P. Werth) for the series "Rheumatologic Skin Disease" published in Annals of Translational Medicine. The article has undergone external peer review.

Conflicts of Interest: The authors have completed the
ICMJE uniform disclosure form (available at http://dx.doi. org/10.21037/atm-20-5552). The series "Rheumatologic Skin Disease" was commissioned by the editorial office without any funding or sponsorship. JFM reports personal fees from Abbbie, personal fees from Dermavant, personal fees from Eli Lilly, personal fees from Novartis, personal fees from Janssen, personal fees from UCB, personal fees from Celgene, personal fees from Sanofi Regeneron, personal fees from Arena, personal fees from Sun Pharma, personal fees from Biogen, personal fees from Pfizer, personal fees from EMD Sorono, personal fees from Avortes, personal fees from Leo Pharm, personal fees from CARA Therapeutics, outside the submitted work. The authors have no other conflicts of interest to declare. 


\section{Page 10 of 12}

Ethical Statement: The authors are accountable for all aspects of the work in ensuring that questions related to the accuracy or integrity of any part of the work are appropriately investigated and resolved

Open Access Statement: This is an Open Access article distributed in accordance with the Creative Commons Attribution-NonCommercial-NoDerivs 4.0 International License (CC BY-NC-ND 4.0), which permits the noncommercial replication and distribution of the article with the strict proviso that no changes or edits are made and the original work is properly cited (including links to both the formal publication through the relevant DOI and the license). See: https://creativecommons.org/licenses/by-nc-nd/4.0/.

\section{References}

1. Amaral ME, Barbuio R, Milanski M, et al. Tumor necrosis factor- $\alpha$ activates signal transduction in hypothalamus and modulates the expression of pro-inflammatory proteins and orexigenic/anorexigenic neurotransmitters. J Neurochem 2006;98:203-12.

2. Postal M, Appenzeller S. The role of Tumor Necrosis Factor-alpha (TNF- $\alpha)$ in the pathogenesis of systemic lupus erythematosus. Cytokine 2011;56:537-43.

3. Chen Y, Sun J, Yang Y, et al. Malignancy risk of anti-tumor necrosis factor alpha blockers: an overview of systematic reviews and meta-analyses. Clin Rheumatol 2016;35:1-18.

4. Atzeni F, Sarzi-Puttini P. Autoantibody production in patients treated with anti-TNF- $\alpha$. Expert Rev Clin Immunol 2008;4:275-80.

5. Brion PH, Mittal-Henkle A, Kalunian KC. Autoimmune skin rashes associated with etanercept for rheumatoid arthritis. Ann Intern Med 1999;131:634.

6. Atzeni F, Talotta R, Salaffi F, et al. Immunogenicity and autoimmunity during anti-TNF therapy. Autoimmun Rev 2013;12:703-8.

7. Colombel JF, Loftus EV Jr, Tremaine WJ, et al. The Safety Profile of Infliximab in Patients with Crohn's Disease: The Mayo Clinic Experience in 500 Patients. Gastroenterology 2004;126:19-31.

8. Aringer M, Smolen JS. Therapeutic blockade of TNF in patients with SLE-Promising or crazy? Autoimmun Rev 2012;11:321-5.

9. Almoallim H, Al-Ghamdi Y, Almaghrabi H, et al. AntiTumor Necrosis Factor- $\alpha$ Induced Systemic Lupus Erythematosus. Open Rheumatol J 2012;6:315-9.

10. Villalta D, Bizzaro N, Bassi N, et al. Anti-dsDNA
Said et al. Anti-TNF-alpha therapy for connective tissue disorders

Antibody Isotypes in Systemic Lupus Erythematosus: IgA in Addition to IgG Anti-dsDNA Help to Identify Glomerulonephritis and Active Disease. PLoS One 2013;8:e71458.

11. De Rycke L, Baeten D, Kruithof E, et al. Infliximab, but not etanercept, induces IgM anti-double-stranded DNA autoantibodies as main antinuclear reactivity: Biologic and clinical implications in autoimmune arthritis. Arthritis Rheum 2005;52:2192-201.

12. De Bandt M, Sibilia J, Le Loët X, et al. Systemic lupus erythematosus induced by anti-tumour necrosis factor alpha therapy: a French national survey. Arthritis Res Ther 2005; 7:R545-51.

13. Weckerle CE, Niewold TB. The unexplained female predominance of systemic lupus erythematosus: Clues from genetic and cytokine studies. Clin Rev Allergy Immunol 2011;40:42-9.

14. Squance ML, Reeves GEM, Bridgman H. The Lived Experience of Lupus Flares: Features, Triggers, and Management in an Australian Female Cohort. Int J Chronic Dis 2014;2014:816729.

15. Tamirou F, Arnaud L, Talarico R, et al. Systemic lupus erythematosus: State of the art on clinical practice guidelines. RMD Open 2018;4:e000793.

16. Jarrett $\mathrm{P}$, Werth VP. A review of cutaneous lupus erythematosus: Improving outcomes with a multidisciplinary approach. J Multidiscip Healthc 2019;12:419-428.

17. Okon LG, Werth VP. Cutaneous lupus erythematosus: Diagnosis and treatment. Best Pract Res Clin Rheumatol 2013;27:391-404.

18. Kim A, O’Brien J, Tseng LC, et al. Autoantibodies and disease activity in patients with discoid lupus erythematosus. JAMA Dermatol 2014;150:651-4.

19. Cortes Hernandez J. SP0138 Anti-TNF Therapy in Systemic Lupus Erythematosus. Ann Rheum Dis 2015;74.

20. Zampieri S, Alaibac M, Iaccarino L, et al. Tumour necrosis factor $\alpha$ is expressed in refractory skin lesions from patients with subacute cutaneous lupus erythematosus. Ann Rheum Dis 2006;65:545-8.

21. Mahto H, Tripathy R, Meher BR, et al. TNF- $\alpha$ promoter polymorphisms (G-238A and G-308A) are associated with susceptibility to Systemic Lupus Erythematosus (SLE) and P. falciparum malaria: a study in malaria endemic area. Sci Rep 2019;9:11752.

22. Crommelin HA, Vorselaars ADM, Van Moorsel CHM, et al. Anti-TNF therapeutics for the treatment of sarcoidosis. Immunotherapy 2014;6:1127-43. 
23. Amber KT, Bloom R, Mrowietz U, et al. TNF- $\alpha$ : A treatment target or cause of sarcoidosis? J Eur Acad Dermatol Venereol 2015;29:2104-11.

24. Ko JM, Gottlieb AB, Kerbleski JF. Induction and exacerbation of psoriasis with TNF-blockade therapy: A review and analysis of 127 cases. J Dermatolog Treat 2009;20:100-8.

25. Tobin AM, Kirby B. TNF $\alpha$ inhibitors in the treatment of psoriasis and psoriatic arthritis. BioDrugs 2005;19:47-57.

26. Varada S, Gottlieb AB, Merola JF, et al. Treatment of coexistent psoriasis and lupus erythematosus. J Am Acad Dermatol 2015;72:253-60.

27. Klein R, Rosenbach M, Kim EJ, et al. Tumor necrosis factor inhibitor-associated dermatomyositis. Arch Dermatol 2010;146:780-4.

28. Iannone F, Scioscia C, Falappone PCF, et al. Use of etanercept in the treatment of dermatomyositis: A case series. J Rheumatol 2006;33:1802-4.

29. Mosca M, Tani C, Filice ME, et al. Tnf-alpha inhibitors in systemic lupus erythematosus. A case report and a systematic literature review. Mod Rheumatol 2015;25:642-5.

30. Kalunian K, Merrill JT. New directions in the treatment of systemic lupus erythematosus. Curr Med Res Opin 2009;25:1501-14.

31. Aringer M, Houssiau F, Gordon C, et al. Adverse events and efficacy of TNF-alpha blockade with infliximab in patients with systemic lupus erythematosus: longterm follow-up of 13 patients. Rheumatology (Oxford) 2009;48:1451-4.

32. Salomon BL, Leclerc M, Tosello J, et al. Tumor necrosis factor $\alpha$ and regulatory $T$ cells in oncoimmunology. Front Immunol 2018;9:444.

33. Billmeier U, Dieterich W, Neurath MF, et al. Molecular mechanism of action of anti-tumor necrosis factor antibodies in inflammatory bowel diseases. World J Gastroenterol 2016;22:9300-13.

34. Lech M, Anders HJ. The pathogenesis of lupus nephritis.J Am Soc Nephrol 2013;24:1357-66.

35. Cantaert T, Baeten D, Tak PP, et al. Type I IFN and TNF $\alpha$ cross-regulation in immune-mediated inflammatory disease: Basic concepts and clinical relevance. Arthritis Res Ther 2010;12:219.

36. Cantaert T, De Rycke L, Mavragani CP, et al. Exposure to nuclear antigens contributes to the induction of humoral autoimmunity during tumour necrosis factor alpha blockade. Ann Rheum Dis 2009;68:1022-9.

37. Kirou KA, Lee C, George S, et al. Activation of the interferon- $\alpha$ pathway identifies a subgroup of systemic lupus erythematosus patients with distinct serologic features and active disease. Arthritis Rheum 2005;52:1491-503.

38. Ramos-Casals M, Brito-Zerón P, Muñoz S, et al. Autoimmune diseases induced by TNF-targeted therapies: Analysis of 233 cases. Medicine (Baltimore) 2007;86:242-51.

39. Keiserman B, Ronchetti MR, Monticielo OA, et al. Concomitance of IgM and IgG anti-dsDNA Antibodies Does Not Appear to Associate to Active Lupus Nephritis. Open Rheumatol J 2013;7:101-4.

40. Charles PJ, Smeenk RJT, De Jong J, et al. Assessment of antibodies to double-stranded DNA induced in rheumatoid arthritis patients following treatment with infliximab, a monoclonal antibody to tumor necrosis factor $\alpha$ : Findings in open-label and randomized placebocontrolled trials. Arthritis Rheum 2000;43:2383-90.

41. Aringer M, Steiner G, Graninger WB, et al. Effects of short-term infliximab therapy on autoantibodies in systemic lupus erythematosus. Arthritis Rheum 2007;56:274-9.

42. Deshmukh US, Bagavant H, Fu SM. Role of antiDNA antibodies in the pathogenesis of lupus nephritis. Autoimmun Rev 2006;5:414-8.

43. Yung S, Chan TM. Anti-DNA antibodies in the pathogenesis of lupus nephritis - The emerging mechanisms. Autoimmun Rev 2008;7:317-21.

44. Olson SW, Lee JJ, Prince LK, et al. Elevated subclinical double-stranded DNA antibodies and future proliferative lupus nephritis. Clin J Am Soc Nephrol 2013;8:1702-8.

45. Aringer $M$, Graninger WB, Steiner G, et al. Safety and efficacy of tumor necrosis factor $\alpha$ blockade in systemic lupus erythematosus: An open-label study. Arthritis Rheum 2004;50:3161-9.

46. Ferraccioli G, Mecchia F, Di Poi E, et al. Anticardiolipin antibodies in rheumatoid patients treated with etanercept or conventional combination therapy: Direct and indirect evidence for a possible association with infections. Ann Rheum Dis 2002;61:358-61.

47. Matsumura R, Umemiya K, Sugiyama T, et al. Anti-tumor necrosis factor therapy in patients with difficult-to-treat lupus nephritis: A prospective series of nine patients. Clin Exp Rheumatol 2009;27:416-21.

48. Dalle Vedove C, Simon JC, Girolomoni G. Drug-induced lupus erythematosus with emphasis on skin manifestations and the role of anti-TNF $\alpha$ agents. J Dtsch Dermatol Ges 2012;10:889-97.

49. Katz U, Zandman-Goddard G. Drug-induced lupus: An 
update. Autoimmun Rev 2010;10:46-50.

50. Vaglio A, Grayson PC, Fenaroli P, et al. Drug-induced lupus: Traditional and new concepts. Autoimmun Rev 2018;17:912-8.

51. Costa MF, Said NR, Zimmermann B. Drug-Induced Lupus due to Anti-Tumor Necrosis Factor a Agents. Semin Arthritis Rheum 2008;37:381-7.

52. Borchers AT, Keen CL, Gershwin ME. Drug-induced lupus. Ann N Y Acad Sci 2007;1108:166-82.

53. Murdaca G, Negrini S, Pellecchio M, et al. Update upon the infection risk in patients receiving TNF alpha inhibitors. Expert Opin Drug Saf 2019;18:219-29.

54. Ali T, Kaitha S, Mahmood S, et al. Clinical use of antiTNF therapy and increased risk of infections. Drug Healthc Patient Saf 2013;5:79-99.

55. Solovic I, Sester M, Gomez-Reino JJ, et al. The risk of tuberculosis related to tumour necrosis factor antagonist therapies: a TBNET consensus statement. Eur Respir J 2010;36:1185-206.

56. Cervera R, Khamashta MA, Font J, et al. Morbidity and mortality in systemic lupus erythematosus during a 10year period: A comparison of early and late manifestations in a cohort of 1,000 patients. Medicine (Baltimore) 2003;82:299-308.

57. Curtis JR, Xi J, Patkar N, et al. Drug-specific and timedependent risks of bacterial infection among patients with rheumatoid arthritis who were exposed to tumor necrosis factor $\alpha$ antagonists. Arthritis Rheum 2007;56:4226-7.

58. Dixon WG, Symmons DPM, Lunt M, et al. Serious infection following anti-tumor necrosis factor $\alpha$ therapy in patients with rheumatoid arthritis: Lessons from interpreting data from observational studies. Arthritis Rheum 2007;56:2896-904.

59. Uppal SS, Hayat SJ, Raghupathy R. Efficacy and safety of infliximab in active SLE: A pilot study. Lupus 2009; 18:690-7.

60. Katz RS, Holt-Daly N, MacDonald PA. Frequent infusion reactions associated with infliximab treatment in patients with polyarthritis related to systemic lupus erythematosus.

Cite this article as: Said JT, Elman SA, Merola JF. Evaluating safety and compatibility of anti-tumor necrosis factor therapy in patients with connective tissue disorders. Ann Transl Med 2021;9(5):430. doi: 10.21037/atm-20-5552
Said et al. Anti-TNF-alpha therapy for connective tissue disorders

Arthritis Rheum 2003;48:S379.

61. Williams CJM, Peyrin-Biroulet L, Ford AC. Systematic review with meta-analysis: Malignancies with anti-tumour necrosis factor- $\alpha$ therapy in inflammatory bowel disease. Aliment Pharmacol Ther 2014;39:447-58.

62. Bernatsky S, Ramsey-Goldman R, Isenberg D, et al. Hodgkin's lymphoma in systemic lupus erythematosus. Rheumatology (Oxford) 2007;46:830-2.

63. Zintzaras E, Voulgarelis M, Moutsopoulos HM. The risk of lymphoma development in autoimmune diseases: A meta-analysis. Arch Intern Med 2005;165:2337-44.

64. Swale VJ, Perrett CM, Denton CP, et al. Etanerceptinduced systemic lupus erythematosus. Clin Exp Dermatol 2003;28:604-7.

65. Stratigos AJ, Antoniou C, Stamathioudaki S, et al. Discoid lupus erythematosus-like eruption induced by infliximab. Clin Exp Dermatol 2004;29:150-3.

66. Vabre-Latre CM, Bayle P, Marguery MC, et al. Worsening of subacute lupus erythematosus induced by infliximab. Ann Dermatol Venereol 2005;132:349-53.

67. Sheth N, Greenblatt D, Patel S, et al. Adalimumabinduced cutaneous lupus. Clin Exp Dermatol 2007;32:593-4.

68. Levine D, Switlyk SA, Gottlieb A. Cutaneous lupus erthyematosus and anti-TNF- $\alpha$ therapy: A case report with review of the literature. J Drugs Dermatol 2010;9:1283-7.

69. Wilkerson E, Hazey MA, Bahrami S, et al. Golimumabexacerbated subacute cutaneous lupus erythematosus. Arch Dermatol 2012;148:1186-90.

70. Norman R, Greenberg RG, Jackson JM. Case reports of etanercept in inflammatory dermatoses. J Am Acad Dermatol 2006;54:S139-42.

71. Yuzaiful M, Yusof M, Wittmann M, et al. 244 Targeted therapy using intradermal injection of etanercept for remission induction in discoid lupus erythematosus (TARGET-DLE): results from a proof-of-concept phase II trial. Rheumatology 2019;58 Available online: https://doi. org/10.1093/rheumatology/kez107.060 